\title{
Sequencing Adapter
}

National Cancer Institute

\section{Source}

National Cancer Institute. Sequencing Adapter. NCI Thesaurus. Code C153363.

A short single-stranded or double-stranded oligonucleotide that is lig ated/added to each fragment of a sequencing library to permit its amplification and subsequent sequencing. 